\title{
Effects of ondansetron, metoclopramide and granisetron on perioperative nausea and vomiting in patients undergone bariatric surgery: a randomized clinical trial
}

\section{Bahador Oshidari}

Shahid Beheshti University of Medical Sciences

Manoochehr Ebrahimian ( $\nabla$ manoochehrebrahimian@sbmu.ac.ir)

Shahid Beheshti University of Medical Sciences https://orcid.org/0000-0003-1286-3308

Negin Ghayebi

Shahid Beheshti University of Medical Sciences School of Medicine

\section{Research Article}

Keywords: Bariatric Surgery, Post-operative nausea or vomiting, Sleeve Gastrectomy, Ondansetron, Metoclopramide, Granisetron

Posted Date: October 19th, 2021

DOI: https://doi.org/10.21203/rs.3.rs-960156/v1

License: (c) (1) This work is licensed under a Creative Commons Attribution 4.0 International License.

Read Full License 


\section{Abstract}

Introduction

Post-operative nausea and vomiting (PONV) is a common problem after bariatric surgeries. In recent years, following the expansion of the number of this type of surgery, special attention has been paid to prevent this phenomenon. Also, several prophylaxis methods have been developed including Enhanced Recovery After Surgery (ERAS) and antiemetics. Nevertheless, PONV has not been completely eliminated and the clinicians are trying to reduce the incidence of PONV, yet.

Methods

After successful ERAS implementation, patients divided into five groups including control and interventional groups. Metoclopramide (MA), Ondansetron (OA), Granisetron (GA) and combination of Metoclopramide and Ondansetron (MO) were used as antiemetics for each groups. The frequency of PONV during first and second days of admission has been recorded.

Results

one hundred thirty patients were enrolled in this study. The MO group showed lower incidence of PONV (46.1\%) in comparison to control group (53.8\%) as well as other groups. Also, the MO group didn't require rescue antiemetic while one-third of control cases used antiemetics (0 vs $34 \%$ ).

Conclusion

Using a combination of metoclopramide and ondansetron is a cost-effective antiemetic regimen for reducing PONV after bariatric surgeries. This combination is more helpful when implemented alongside ERAS protocols.

\section{Introduction}

Post-operative nausea and vomiting (PONV) is one of the leading causes of patient morbidity after laparoscopic bariatric surgeries. A wide variety of complications related to PONV has been described, such as prolonged length of stay (LOS) in hospital, unnecessary readmissions, delay in oral intake, and bad experience for patients [1]. Although several antiemetic regimens have been tried so far in different studies, the incidence of PONV is not significantly lowered, and it seems that it is impossible to totally eliminate it. On the other hand, the implementation of Enhanced Recovery After Surgery (ERAS) has greatly reduced the incidence of PONV and LOS. [2]. Therefore, a combination of ERAS and multiple antiemetic regimens is currently used to reduce the incidence of PONV. Nevertheless, the optimal regimen has not been found yet, and many trials are conducting to find out the best antiemetic regimen.[1, 2]. In this randomized clinical trial, we compare four different combined and single regimens alongside the implementation of ERAS to show which regimen is more effective. 


\section{Methods}

After being approved by Iran National Committee for Ethics in Biomedical Research (IR.SBMU.MSP.REC.1399.784), a randomized clinical controlled trial was started with 130 patients in five groups. All the patients were proper candidates for laparoscopic bariatric surgeries, and structured informed consent was obtained from all participants.

All the operations were performed by board certified advanced laparoscopic surgeons in a minimally invasive educational center. All bariatric surgery was performed in our standardized institutional protocols as well as preoperative and postoperative care. Sleeve Gastrectomy (SG) surgeries were performed by using 44Fr Tubes.

To calculate the sample size in the clinical trial, we used the ANCOVA method with web-based tools. Twenty-six patients were estimated for each group [3]. Patients were divided into five groups:

Group 1: Patients who did not receive any antiemetic during hospitalization (NA).

Group 2: Patients receiving metoclopramide alone (MA).

Group 3: Patients who received ondansetron only. (OA)

Group 4: Patients receiving a combination of metoclopramide and ondansetron (MO).

Group 5: Patients who received granisetron alone (GA).

All patients were undergone ERAS protocols. In cases where the patient had PONV (including Group 1), intravenous Metoclopramide $0.2 \mathrm{mg}$ (Stat and $\mathrm{BiD}$ ) was used as a rescue antiemetic.

To reduce the incidence of bias and confounding factors, all anesthetics and antiemetics used were provided from the same brand for each drug (see Appendix).

Patients with severe or moderate gastritis or duodenitis on esophagogastroduodenoscopy were excluded from the study, but patients with mild gastritis or positive rapid urease test on endoscopy, were treated for two weeks with three drugs of pentazole, amoxicillin, and metronidazole, and if the respiratory urease test was negative, they were included in the study and in refractory cases of $\mathrm{H}$. Pylori, they were excluded. According to the American Society of Anesthesiologists (ASA) classification [4], patients with severe respiratory or cardiovascular problems (ASA III or higher) or a history of gastric or small bowel surgery were also excluded. Patients who underwent simultaneous cholecystectomy with bariatric surgery were also excluded (Figure 1).

\section{Statistical Analysis}

Information about demographic, clinical, and paraclinical variables, as well as intraoperative data, was 
information, digital scales and meters, as well as obtaining a direct history of the patient were used. The duration of anesthesia was calculated in minutes from the moment of induction of anesthesia to the moment the patient regained consciousness and transferred to Post-Anesthesia Care Unit (PACU). Nausea and vomiting were also recorded by taking a history or direct observation.

Descriptive and analytical functions were used to analyze the information. Frequency, mean and median and standard deviation were used for variables of age, sex, weight, height, body mass index, incidence of nausea and vomiting, etc. (see Table1-6). To investigate the significant relationship between the variables, Correspondence Analysis was used. Partial correlation with Pearson formula was also used to investigate the confounding effect of other variables and their effect on nausea and vomiting.

Table 1

Demographic data

\begin{tabular}{|c|c|}
\hline Variable & Numbers (Percent) \\
\hline Total cases & $130(100 \%)$ \\
\hline Age & $36.3(19-59)$ \\
\hline \multicolumn{2}{|l|}{ Gender } \\
\hline Men & $31(23.8 \%)$ \\
\hline Women & $99(76.2 \%)$ \\
\hline Weight (average, kilograms) & 121.36 \\
\hline Height (average, centimeters) & 164.6 \\
\hline Body mass index (average, $\frac{\mid \text { varvec } k \mid \text { varvec } g}{\mid \text { varvecm }{ }^{2}}$ ) & $44.6, \mathrm{Std}=5.22$ \\
\hline
\end{tabular}


Table 2

preoperative clinical infromation

\begin{tabular}{|ll|}
\hline Numbers (percent) & Variable \\
\hline $83(63.8 \%)$ & Underlying diseases \\
\hline $19(14.6 \%)$ & No UD $^{*}$ \\
\hline $14(10.8 \%)$ & Typothyroidism \\
\hline $14(10.8 \%)$ & Hypertension \\
\hline $13(10 \%)$ & Two concurrent UD \\
\hline $3(2.3 \%)$ & Equal or more than three UD \\
\hline History of previous surgery (general anesthesia) \\
\hline $55(42.3 \%)$ & No surgeries \\
\hline $48(36.9 \%)$ & One \\
\hline $19(14.6 \%)$ & Two \\
\hline $8(6.1 \%)$ & Three or more \\
\hline$*$ UD = Underlying disease(s) \\
\hline
\end{tabular}


Table 3

preoperative paraclinical information

\begin{tabular}{|ll|}
\hline Variable & Numbers (percent) \\
\hline Upper endoscopy findings & \\
\hline Normal & $80(61.5 \%)$ \\
\hline Antral gastritis & $21(16.2 \%)$ \\
\hline Erosive gastritis & $13(10 \%)$ \\
\hline Duodenal erythema & $5(3.8 \%)$ \\
\hline Duodenal diverticula & $2(1.5 \%)$ \\
\hline Biliary gastritis & $4(3.1 \%)$ \\
\hline Esophagitis & $4(3.1 \%)$ \\
\hline Stomach polyp(s) & $1(0.8 \%)$ \\
\hline Respiratory urease test & \\
\hline Negative & $100(76.9 \%)$ \\
\hline Positive & $28(21.5 \%)$ \\
\hline Fatty liver (based on abdominal ultrasonography) & $20(15.4)$ \\
\hline No fatty liver & $41(31.5)$ \\
\hline Grade 1 & $49(37.7)$ \\
\hline Grade 2 & $20(15.4)$ \\
\hline Grade 3 & \\
\hline
\end{tabular}


Table 4

intra and postoperative information

\begin{tabular}{|ll|}
\hline Variable & Numbers (percent) \\
\hline Surgery type & \\
\hline Sleeve gastrectomy & $115(88.5)$ \\
\hline Mini-bypass & $15(11.5)$ \\
\hline Average time of anesthesia & $141.8(75305 \mathrm{QQ})$ \\
\hline Average dose of anesthetics & \\
\hline Fentanyl & 195.16 \\
\hline Midazolam & 2.28 \\
\hline Lidocaine & 91.9 \\
\hline Thiopental & 498.3 \\
\hline Atracurium & 63.05 \\
\hline Pain pumps & $18(13.8)$ \\
\hline
\end{tabular}

Table 5

incidence of PONV in the perioperative period

\begin{tabular}{|llllll|}
\hline \multicolumn{5}{|c|}{ Before oral intake (day 1) } & After oral intake (day 2) \\
\hline Regimen & Nausea & Vomiting & Nausea & Vomiting & Rescue usage \\
\hline Control & 11 & 6 & 7 & 3 & 9 \\
\hline MA & 12 & 8 & 7 & 2 & 2 \\
\hline OA & 10 & 5 & 9 & 2 & 6 \\
\hline MO & 7 & 6 & 2 & 0 & 0 \\
\hline GA & 9 & 7 & 6 & 3 & 8 \\
\hline
\end{tabular}


Table 6

total incidence of PONV during admission

\begin{tabular}{|ll|}
\hline Study group & Total incidence of PONV (including days 1 and 2) \\
\hline Control group & 53.8 \\
\hline MA & 53.8 \\
\hline OA & 61.5 \\
\hline MO & 46.1 \\
\hline GA & 61.5 \\
\hline
\end{tabular}

\section{Results}

\section{Demographics}

One hundred thirty morbid obese patients were enrolled in this study from March 2021 to August 2021. They were divided into five groups of 26 participants. All demographic information is displayed in the Table 1.

\section{Clinical and paraclinical findings}

Information like the presence of underlying diseases, and history of previous episodes of anesthesia, esophagogastroduodenoscopy results, H-Pylori, and fatty liver grade has been described in Tables 2-3.

\section{Perioperative variables}

Table 4 indicates the distribution of the type of surgery, the average time of general anesthesia, and the mean dosage of anesthetic medications as well as usage of Patient-Controlled Anesthesia devices.

Also, the findings related to the incidence of nausea and vomiting in the postoperative period on days 1 and $2 \square$ are presented in Table 5. It can be quickly realized that the incidence of nausea and vomiting is generally higher on the first day after surgery than on the second day. On the other hand, the highest incidence of nausea on the first day (before starting a liquid diet) was in the metoclopramide group ( $\mathrm{n}=$ 12 ) and the lowest was in the metoclopramide and ondansetron group $(n=7)$. The incidence of vomiting on the first day has a similar distribution; Thus, the highest level is in the metoclopramide group alone ( $\mathrm{n}$ $=8$ ) and the lowest level is in the ondansetron group alone $(n=5)$. On the second day, the lowest incidence of nausea and vomiting was in the metoclopramide and ondansetron groups (Table 5).

\section{Results analysis}

Table 6 shows the final incidence of nausea and vomiting during the entire hospital stay. As it turns out, the lowest incidence of nausea or vomiting is in the group that used metoclopramide and ondansetron together $(46.1 \%)$. The highest incidence of nausea or vomiting is related to ondansetron or granisetron Loading [MathJax]/jax/output/CommonHTML/jax.js lationship between each of the variables that may be 
confusing the results of nausea and vomiting is indicated. In almost all fields, there is no significant relationship between age, sex, body mass index, duration of operation, types of drugs used during anesthesia, underlying diseases and previous surgery, and endoscopic findings with nausea and vomiting. A lower p-value (0.01) in variables such as duration of operation, usage of fentanyl, and thiopental drugs on the first day, means that these variables may have an effect on the incidence of nausea, but this change is not significant (see $p$ values in Table 7)

Table 7

correlation between other variables in the incidence of PONV

\begin{tabular}{|c|c|c|c|c|}
\hline \multirow[b]{2}{*}{ variable } & \multicolumn{2}{|c|}{ Before oral intake (day 1) } & \multicolumn{2}{|c|}{ After oral intake (day 2) } \\
\hline & $\begin{array}{l}\text { Nausea ( } p \\
\text { value) }\end{array}$ & $\begin{array}{l}\text { Vomiting ( } p \\
\text { value) }\end{array}$ & $\begin{array}{l}\text { Nausea ( } p \\
\text { value) }\end{array}$ & $\begin{array}{l}\text { Vomiting (p } \\
\text { value) }\end{array}$ \\
\hline Age & 0.740 & 0.075 & 0.785 & 0.192 \\
\hline Gender $^{1}$ & 0.474 & 0.027 & 0.501 & 0.285 \\
\hline$B M I$ & 0.974 & 0.358 & 0.162 & 0.477 \\
\hline Operation time & $0.01 *$ & 0.905 & 0.933 & 0.137 \\
\hline Fentanyl & 0.065 & 0.515 & 0.562 & 0.324 \\
\hline Midazolam & 0.225 & 0.427 & 0.265 & 0.690 \\
\hline Lidocaine & 0.186 & 0.099 & 0.046 & 0.000 \\
\hline thiopental & 0.080 & 0.948 & 0.187 & 0.991 \\
\hline Underlying disease $^{1}$ & 0.210 & 0.591 & 0.298 & 0.246 \\
\hline $\begin{array}{l}\text { Number of previous } \\
\text { surgeries }^{1}\end{array}$ & 0.591 & 0.260 & 0.602 & 0.375 \\
\hline Endoscopy findings ${ }^{1}$ & 0.029 & 0.031 & 0.723 & 0.540 \\
\hline \multicolumn{5}{|c|}{$1=$ this variable is qualitative and Correspondence Analysis was used to examine its correlation. } \\
\hline$\star=$ negative correlatior & arson's co & equal to & & \\
\hline
\end{tabular}

\section{Discussion}

Our results indicate lower episodes of PONV in the MO group. Also, single drug regimens showed equal or higher in comparison to controlled group. Granisetron is a serotonin-receptor antagonist (5HTRA) and has a great reputation for treating nausea/vomiting after chemotherapy or radiotherapy. For this reason, little information is available about the effect of this drug on PONV after bariatric surgery. The only study in this area showed a $30 \%$ incidence of PONV after bariatric surgery. Also combining granisetron with Loading [MathJax]/jax/output/CommonHTML/jax.js 5$]$. However, in our study GA couldn't do this and the incidence 
of PONV was $61.5 \%$. Ondansetron is another 5HTA that, unlike granisetron, has been studied many times in PONV. Overall incidence of OA has been reported about $50 \%$ which is a little lower than our result [6-9]. On the other hand, there are a lot of data from combining ondansetron with other medications such as Aprepitant[10] and Dexamethasone[11] that could lower PONV more than OA. Our ondansetron combination was the $\mathrm{MO}$ group. Metoclopramide belongs to the group of medications known as dopamine-receptor antagonists and is widely used as an antiemetic. However, metoclopramide alone is used as an antiemetic for PONV prophylaxis[12], but it is seems that several clinicians use it as a rescue antiemetic[13]. Interestingly, although these two drugs alone are well known, the combination of the two has been less commonly used in PONV after bariatric surgery (see Appendix). More interestingly, this combination has been less used in other laparoscopic surgeries like cholecystectomy [14]. on the other hand, in our study, MO groups showed significantly lower incidence of PONV among other groups (46.1\%). Given that, these two drugs have two different mechanism of action it seems that this combination can be a useful regimen for controlling PONV.

\section{Conclusion}

Using a combination of metoclopramide and ondansetron is a cost-effective antiemetic regimen for reducing PONV after bariatric surgeries. This combination is more helpful when implemented alongside ERAS protocols. However, there is a scarcity of randomized clinical trials using this combination, and further studies with a high volume of participants can help clinicians to improve their knowledge in PONV prophylaxis.

\section{Declarations}

\section{Contributions}

BO developed the concept and performed the surgery. ME reviewed and analyzed the cases and co-wrote the paper. NG reviewed and co-wrote the paper.

\section{Conflict of interest}

All of the authors declare that they haven't any conflict of interest.

\section{Funding}

None. No funding to declare.

\section{Consent for publication}

An informed consent was taken from the patient.

\section{Ethical Approval}


This topic is approved by Iran National Committee for Ethics in Biomedical Research (IR.SBMU.MSP.REC.1399.784).

\section{References}

1. Fathy M, Abdel-Razik MA, Elshobaky A, Emile SH, El-Rahmawy G, Farid A, et al. Impact of pyloric injection of magnesium sulfate-Lidocaine mixture on postoperative nausea and vomiting after laparoscopic sleeve Gastrectomy: a randomized-controlled trial. Obesity surgery. 2019;29(5):1614-23.

2. Parisi A, Desiderio J, Cirocchi R, Trastulli S. Enhanced Recovery after Surgery (ERAS): a Systematic Review of Randomised Controlled Trials (RCTs) in Bariatric Surgery. Obes Surg. 2020;30(12):5071-85.

3. Borm GF, Fransen J, Lemmens WA. A simple sample size formula for analysis of covariance in randomized clinical trials. Journal of clinical epidemiology. 2007;60(12):1234-8.

4. Wolters $\mathrm{U}$, Wolf $\mathrm{T}$, Stützer $\mathrm{H}$, Schröder T. ASA classification and perioperative variables as predictors of postoperative outcome. British journal of anaesthesia. 1996;77(2):217-22.

5. Moussa AA, Oregan PJ. Prevention of postoperative nausea and vomiting in patients undergoing laparoscopic bariatric surgery--granisetron alone vs granisetron combined with dexamethasone/droperidol. Middle East J Anaesthesiol. 2007;19(2):357-67.

6. Mendes MN, Monteiro RdS, Martins FANdC. Prophylaxis of postoperative nausea and vomiting in morbidly obese patients undergoing laparoscopic gastroplasties: a comparative study among three methods. Revista brasileira de anestesiologia. 2009;59:570-6.

7. Benevides ML, de Souza Oliveira SS, de Aguilar-Nascimento JE. The combination of haloperidol, dexamethasone, and ondansetron for prevention of postoperative nausea and vomiting in laparoscopic sleeve gastrectomy: a randomized double-blind trial. Obesity surgery. 2013;23(9):1389-96.

8. Bamgbade OA, Oluwole O, Khaw RR. Perioperative antiemetic therapy for fast-track laparoscopic bariatric surgery. Obesity surgery. 2018;28(5):1296-301.

9. Ziemann-Gimmel P, Goldfarb A, Koppman J, Marema R. Opioid-free total intravenous anaesthesia reduces postoperative nausea and vomiting in bariatric surgery beyond triple prophylaxis. British journal of anaesthesia. 2014;112(5):906-11.

10. Sinha AC, Singh PM, Williams NW, Ochroch EA, Goudra BG. Aprepitant's prophylactic efficacy in decreasing postoperative nausea and vomiting in morbidly obese patients undergoing bariatric surgery. Obesity surgery. 2014;24(2):225-31.

11. Bataille A, Letourneulx J-F, Charmeau A, Lemedioni P, Léger P, Chazot T, et al. Impact of a prophylactic combination of dexamethasone-ondansetron on postoperative nausea and vomiting in Loading [MathJax]/jax/output/CommonHTML/jax.js ic sleeve gastrectomy during closed-loop propofol- 
remifentanil anaesthesia: A randomised double-blind placebo-controlled study. European Journal of Anaesthesiology (EJA). 2016;33(12):898-905.

12. Talebpour M, Omrani NG, Imani F, Moharari RS, Pourfakhr P, Khajavi MR. Comparison effect of promethazine/dexamethasone and metoclopramide/dexamethasone on postoperative nausea and vomiting after laparascopic gastric placation: a randomized clinical trial. Anesthesiology and pain medicine. 2017;7(4).

13. Naeem Z, Chen IL, Pryor AD, Docimo S, Gan TJ, Spaniolas K. Antiemetic Prophylaxis and Anesthetic Approaches to Reduce Postoperative Nausea and Vomiting in Bariatric Surgery Patients: a Systematic Review. Obesity surgery. 2020;30(8).

14. Wu SJ, Xiong XZ, Cheng TY, Lin YX, Cheng NS. Efficacy of ondansetron vs. metoclopramide in prophylaxis of postoperative nausea and vomiting after laparoscopic cholecystectomy: a systematic review and meta-analysis. Hepatogastroenterology. 2012;59(119):2064-74.

\section{Figures}




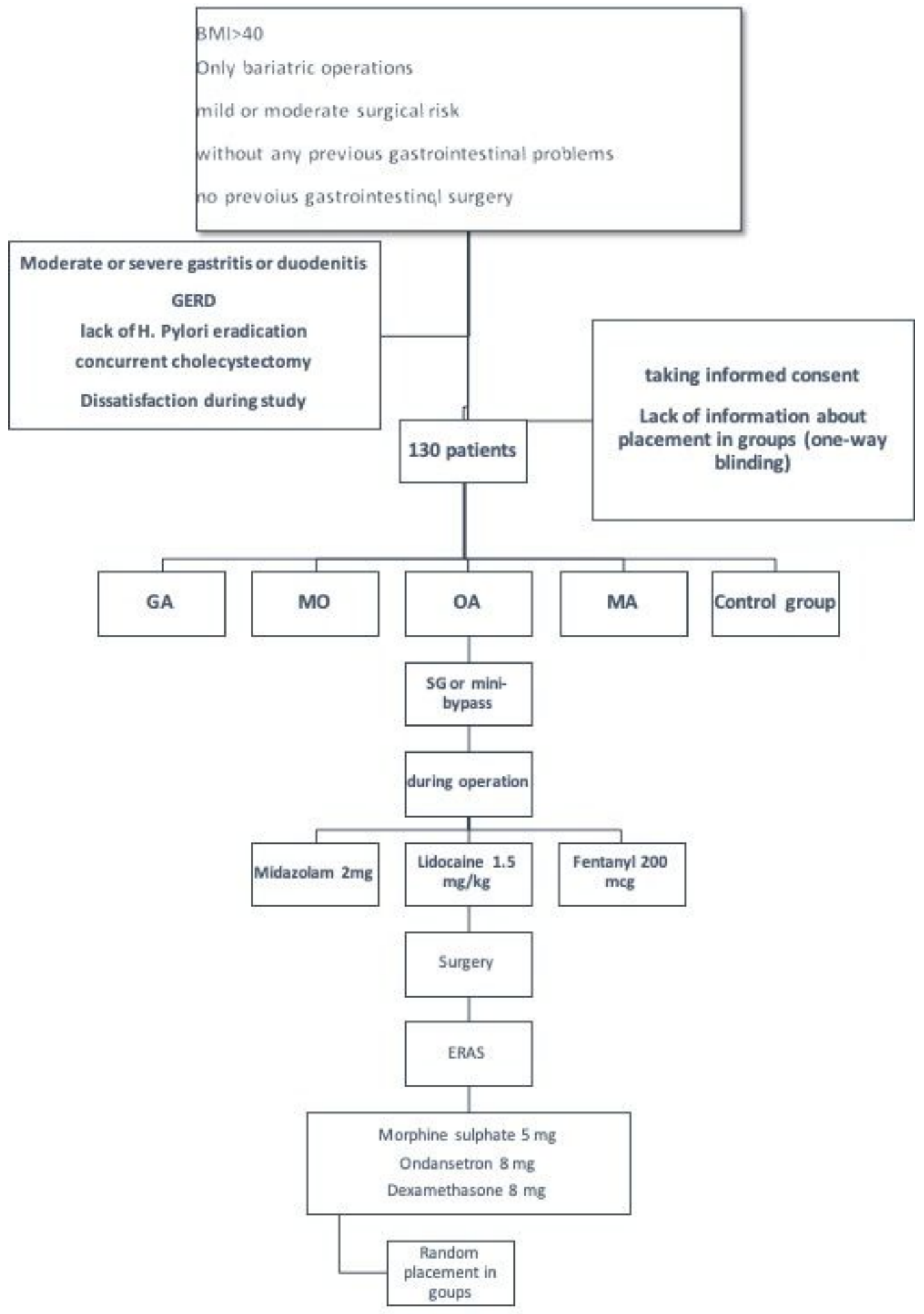

\section{Figure 1}

The Enrollment Flow Chart of the Patients 